\title{
EL DESARROLLO DEL PERFIL DEL TEACHERPRENEUR O PROFESOR- EMPRENDEDOR EN EL CURRÍCULUM DEL GRADO DE EDUCACIÓN PRIMARIA: ¿UN CONCEPTO DE MODA O UNA REALIDAD?
}

\author{
Arantza Arruti Gómez \\ Universidad de Deusto
}

\begin{abstract}
RESUMEN: En el siguiente artículo profundizamos en el concepto teacherpreneur o profesor-emprendedor. Nos aproximamos a él desde la óptica del maestro de Educación Primaria, concretamente desde la formación que durante la realización de los estudios del grado en Educación Primaria se presupone que recibe el futuro maestro de Educación Primaria. Comenzaremos incidiendo en la importancia del emprendimiento y de la educación en emprendimiento para a continuación indicar las características y competencias propias del teacherpreneur; las que se subrayan desde la Comisión Europea así como las que se demandan desde las leyes y decretos que rigen nuestro sistema educativo. El artículo finaliza con una serie de recomendaciones sobre las competencias que el futuro maestro de Educación Primaria debería desarrollar durante la realización de sus estudios del grado en Educación Primaria con la finalidad de lograr el desarrollo de la competencia emprendedora. Sin duda alguna, una competencia propia del teacherpreneur, la persona que hará las funciones de facilitador y guía de las futuras generaciones de emprendedores.
\end{abstract}

PALABRAS CLAVE: Teacherpreneur, iniciativa emprendedora, educación emprendedora, innovación.

\section{THE DEVELOPMENT OF THE PROFILE OF THE TEACHERPRENEUR THROUGH THE PRIMARY EDUCATION DEGREE CURRICULUM: A TRENDY CONCEPT OR A REALITY?}

\footnotetext{
ABSTRACT: In the following article we will delve into the concept teacherpreneur. We will analyze it from the perspective of primary school teachers' profile. We will particularly take into account the curriculum of the future Primary teachers' degree. We will start stressing the importance of entrepreneurship and entrepreneurship education. After that we will indicate the characteristics and competencies of teacherpreneur; those which are
} 
underlined by the European Commission as well as those demanded by the laws and decrees that govern our education system. The article ends with a series of recommendations concerning the competencies that future Primary Education teachers should develop during their studies (Degree of Primary Education) in order to develop the entrepreneurial competence. Doubtlessly, a competence of a teacherpreneur itself, the person who will be the facilitator and guide of future entrepreneurs generations.

KEYWORDS: Teacherpreneur, initiative, entrepreneurship education, innovation.

Recibido: 30/04/2015

Aceptado: 24/07/2015

Correspondencia: Arantza Arruti Gómez, Universidad de Deusto, Facultad de Psicología y Educación, Departamento de Didáctica y Desarrollo Curricular, Avda. de las Universidades 24, 48007 Bilbao (Bizkaia, España). Email: aarruti@deusto.es.

\section{INTRODUCCIÓN}

Que el término emprender así como sus palabras derivadas y términos afines son conceptos actuales y "de moda" no es algo nuevo, de hecho, cada vez son más los estudios e investigaciones realizadas acerca del emprendimiento (social), la persona emprendedora, el espíritu emprendedor, la iniciativa emprendedora, la competencia emprendedora, la competencia aprender a emprender y un largo etcétera. Tanto es así que incluso no es extraño encontrarnos estos términos, u otros afines, en el contexto educativo.

Es por ello por lo que en este artículo nos proponemos, entre otros, dejar constancia de que ser emprendedor/a es algo más que montar un negocio, que ser emprendedor/a no es sólo propio de los hombres y mujeres de negocios, que educar la iniciativa emprendedora y desarrollar la competencia emprendedora es posible, y que emprender está principalmente relacionado con nuestra actitud ante la vida, ante la forma de hacer las cosas y ante la manera de enfrentarnos a los retos y desafíos que ésta nos va marcando. A todo ello tendremos que hacer frente de manera más creativa e innovadora que de costumbre si queremos avanzar en un mundo tan cambiante y competitivo como el nuestro.

Y es desde ese punto de vista desde el cual nos vamos a aproximar a la figura del teacherpreneur o profesor-emprendedor (en adelante, teacherpreneur) y a las competencias y características personales que le son propias y le caracterizan. También abordaremos la idea de que emprender es posible, no sólo cuando nos lanzamos a crear nuevos negocios, sino cuando desde nuestro perfil formador y educador actuamos como profesionales emprendedores, con unos rasgos y un modo de actuar determinados.

En esa línea, en este artículo, nos detendremos y analizaremos las competencias propias del teacherpreneur, concretamente aquellas que, a nuestro parecer, y siguien- 
do fundamentalmente las ideas trabajadas por la Comisión Europea en la guía Educación en emprendimiento: Guía del educador (Comisión Europea, 2014), deberían desarrollar los estudiantes del grado en Educación Primaria (es decir, los futuros maestros ${ }^{1}$ de Educación Primaria). Así mismo, nos centraremos también en las metodologías más adecuadas y eficaces que se utilizan y/o se podrían utilizar en las instituciones de enseñanza superior con el fin de facilitar el desarrollo de la iniciativa emprendedora entre los futuros maestros. Todo ello a la luz de las diferentes leyes que guían nuestro sistema educativo y que demandan un perfil profesional adaptado a la realidad del momento.

\section{JUSTIFICACIÓN DE LA NECESIDAD DE UNA EDUCACIÓN EN EMPRENDIMIENTO EN}

\section{LA EDUCACIÓN SUPERIOR}

En enero de 2013, la Comisión Europea adoptó el Plan de Acción sobre Emprendimiento 2020 (en adelante, Plan 2020), que se define como "un programa de acciones conjuntas decisivas para liberar el potencial del emprendimiento en Europa, eliminar los obstáculos existentes y revolucionar la cultura del emprendimiento" (Comisión Europea, 2013a: 5). En este Plan 2020 se proponen, entre otras, las siguientes áreas de intervención inmediatas: educar y formar en materia de emprendimiento, y dinamizar la cultura del emprendimiento en Europa, creando para ello una nueva generación de emprendedores.

Y es en este mismo Plan 2020 en el que se deja claro que:

...acaben o no fundando empresas o empresas sociales, los jóvenes que se benefician del aprendizaje del emprendimiento desarrollan los conocimientos empresariales y aptitudes y actitudes esenciales, como la creatividad, la iniciativa, la tenacidad, el trabajo en equipo, el conocimiento del riesgo y el sentido de la responsabilidad. Esa es la mentalidad emprendedora que les ayuda a transformar las ideas en actos y también aumenta considerablemente la empleabilidad (Comisión Europea, 2013a: 6).

Es por ello que, como consecuencia del Plan 2020, se llevaron a cabo una serie de acciones, entre las que destacamos la publicación en el año 2014 de la guía Educación en emprendimiento. Guía del Educador. Es en dicha Guía en la que, entre otros, se reconoce a la educación emprendedora como una herramienta que puede ayudar a los jóvenes a ser más emprendedores y a desarrollar un conjunto de competencias aplicables a todos los ámbitos de la vida (y no sólo a gestionar un negocio). De hecho, ya en noviembre de 2012, la Comisión Europea publicó la Comunicación Un nuevo concepto de educación: invertir en las aptitudes para lograr mejores resultados socioeconómico (Comisión Europea, 2013b) en la que se hacía hincapié en la necesidad de desarrollar competencias transversales como el espíritu emprendedor, el pensamiento crítico, la iniciativa, la resolución de problemas o el trabajo colaborativo.

En esa misma línea, no es de extrañar que uno de los principales objetivos de la Comisión Europea sea promover la educación emprendedora al tiempo que se resalta su importancia en todos los niveles educativos. No en vano, este tipo de educación

1. En adelante se utilizará el término maestro para referirnos al colectivo de maestros y maestras. 
está dirigida a preparar a las personas para ser personas responsables y emprendedoras, ayudándoles a desarrollar las habilidades, conocimientos y actitudes necesarias para alcanzar sus propios objetivos.

De hecho, ya en el año 2006, en su Comunicación sobre la aplicación del Programa Comunitario de Lisboa. Fomentar la mentalidad emprendedora mediante la educación y la formación (en adelante, Programa Comunitario de Lisboa), la Comisión Europea reconoce que "el emprendimiento debería adquirirse a través del aprendizaje permanente y aboga por una enseñanza de esta competencia empezando desde la escuela primaria hasta la universidad, incluyendo la formación profesional secundaria (formación profesional inicial) y los centros técnicos superiores" (Comisión Europea, 2006: 4). Y en lo que respecta a la enseñanza superior, en esa misma Comunicación, se establece que: "las universidades e institutos técnicos deberían integrar el emprendimiento, desglosado en varias asignaturas, como una parte importante de su plan de estudios, y exigir o recomendar a los estudiantes que sigan los cursos sobre emprendimiento" (Comisión Europea, 2006: 9). En ese sentido, la Comisión considera que integrar el emprendimiento en diversas asignaturas de sus programas de estudio puede ofrecer un valor añadido a los distintos grados universitarios.

Aún así, en el año 2008, el grupo de expertos que participó en la redacción del Informe Final sobre la Iniciativa Emprendedora en la Enseñanza Superior, especialmente en Estudios no Empresariales (Comisión Europea, 2008) (en adelante, Informe Final sobre la Iniciativa Emprendedora) todavía seguía afirmando que la iniciativa emprendedora aún no estaba suficientemente incorporada en los planes de estudios de la enseñanza superior. Es más, según los datos de que se disponían la mayoría de cursos sobre competencias emprendedoras estaban incluidos en los estudios de empresariales y económicas (este es el caso, por ejemplo, de España). Fue por ello por lo que se incidió en que el verdadero reto consistía en crear enfoques interdisciplinares, tanto en lo que a ámbitos de estudio se refiere como en lo que a diversidad de perfiles y procedencia de estudiantes afecta, pudiendo Ilegar a reunir a estudiantes procedentes de distintas facultades y de distintos ámbitos de estudio.

Es por ello que desde el Plan 2020 se insiste en que hay que:

....animar a los centros de enseñanza a que sean más emprendedores en sus enfoques más amplios, para garantizar que desarrollen y vivan una cultura del emprendimiento y la innovación a través de sus cometidos, liderazgo, participación de los interesados, planes de estudio y resultados del aprendizaje, porque nos encontramos en un momento en que los centros de enseñanza superior se convierten en un componente activo de las políticas de innovación de los Estados miembros y de la UE (Comisión Europea, 2013a: 7).

Y es que, según Gibb, Haskins y Robertson (2013) o Taatila (2010), la universidad debe hacerse más emprendedora y es por ello que la Comisión, en su Plan 2020 invita a los Estados miembros a que, entre otros: "garanticen que la competencia clave «emprendimiento» esté integrada en los planes de estudios de la enseñanza primaria y secundaria, la formación profesional, la enseñanza superior y la educación de adultos antes del final de 2015" (Comisión Europea, 2013a: 8). Y no sólo eso, la 
Comisión se sigue reafirmando en que "se precisa un cambio radical de la cultura europea que cambie su noción de emprendimiento" (Comisión Europea, 2013a: 30).

Llegados a este punto merece la pena rescatar algunas de las soluciones propuestas en el Informe Final sobre la Iniciativa Emprendedora en lo que a las instituciones de enseñanza superior se refiere: establecer una estrategia y un plan de acción para la enseñanza y la investigación en materia de iniciativa emprendedora, ofrecer a todos los estudiantes de primer ciclo la posibilidad de seguir durante su primer año actividades o módulos introductorios obligatorios sobre emprendimiento que influyan en su mentalidad desde el principio y les sensibilice respecto de la posibilidad de ser emprendedor. Además, dar a todos los estudiantes la oportunidad de asistir a seminarios y conferencias sobre esta temática. Así mismo, se propone que las instituciones de enseñanza superior ofrezcan una gama de cursos (optativos, extracurriculares, concursos, etc.), a no limitarse a un modelo particular de enseñanza y a ser más flexibles en el diseño de los cursos (ofertar estudios a tiempo completo y parcial, organizar cursos intensivos, proponer periodos de prácticas y acreditar aprendizajes tanto formales como no formales).

\section{El EMPRENDIMIENTO: COMPETENCIA CLAVE PARA EL DESARROLLO INTEGRAL DE}

\section{LAS PERSONAS Y EN ESPECIAL DE LOS FUTUROS MAESTROS}

En el año 2000, tras la Declaración de Bolonia, surgió un proyecto piloto denominado Proyecto "Tuning - Sintonizar las estructuras educativas de Europa" (González y Wagenaar, 2003) que abordaría varias de las líneas de acción señaladas en Bolonia. Entre sus objetivos estaba el de determinar los puntos de referencia para el establecimiento, a escala europea, de las competencias genéricas y específicas para cada disciplina, en una serie de campos temáticos. La competencia "iniciativa y espíritu emprendedor" fue una de las 30 competencias genéricas resultantes de este Proyecto y que hoy en día es de referencia no sólo para Europa sino también para América Latina. Pero, ¿a qué nos referimos cuando hablamos de emprendimiento, espíritu emprendedor o iniciativa emprendedora (así como otros términos afines)? En primer lugar, es necesario aclarar la no existencia de una definición consensuada sobre lo que es emprender o lo que la competencia emprendedora significa (Alemany, Alvarez, Planellas y Urbano, 2011; Alemany y Planellas, 2011; Marina, 2010). De hecho, no sólo la Comisión Europea sino otros muchos expertos utilizan indistintamente cualquiera de los términos arriba mencionados para referirse a la competencia emprendedora. Aun así, existe cierto consenso a la hora de establecer las principales características con las que identificamos a la persona emprendedora, como podremos ver más adelante.

Volviendo al término que nos ocupa, ya en el año 2003, la Comisión de las Comunidades Europeas definió la iniciativa emprendedora como una actitud, una forma de pensar o mentalidad a la vez que un proceso para crear actividad (económica). Este proceso incluye motivación, toma de riesgos, creatividad, innovación, capacidad para identificar oportunidades, luchar por ellas, producir valor, inducir cambios, asumir responsabilidades, establecer objetivos y cumplirlos. (Comisión de las Comunidades Europeas, 2003 y Comisión Europea, 2004). 
Es también en el año 2003 cuando con el objetivo de servir de ayuda al futuro maestro, se edita la guía para el profesor denominada El espíritu emprendedor. Motor de cambio. En ella se establece que:

... las personas dotadas de espíritu emprendedor poseen la capacidad de innovar; tienen voluntad de probar cosas nuevas o hacerlas de manera diferente... el que llamamos espíritu emprendedor conlleva un aspecto mucho más amplio de actitudes positivas... supone querer desarrollar capacidades de cambio, experimentar con las ideas propias y reaccionar con mayor apertura y flexibilidad. El espíritu emprendedor presenta una doble faceta. Por un lado... supone saber lanzar nuevos proyectos con autonomía, capacidad de asumir riesgo, con responsabilidad, con intuición, con capacidad de proyección al exterior y con capacidad de reaccionar y resolver los problemas. Por otro lado, también supone saber llevar a cabo proyectos de otros con el mismo espíritu de innovación, responsabilidad y autonomía. Educar el espíritu emprendedor exige formar a los alumnos en la responsabilidad, el compromiso, el esfuerzo, la dedicación, la perseverancia y el espíritu de trabajo (Dirección General de Política de la Pequeña y Mediana Empresa, 2003: 10).

Por su parte, en el Programa Comunitario de Lisboa, publicado en el 2006, se entiende por emprendimiento (entrepreneurship):

... la habilidad de un individuo para convertir ideas en actos. Incluye la creatividad, la innovación y la asunción de riesgos, así como la habilidad para planificar y gestionar proyectos destinados a lograr objetivos. Es un apoyo para todos en la vida cotidiana del hogar y en la sociedad, hace que el empleado sea más consciente del contexto de su trabajo y más capaz de aprovechar sus oportunidades, es la base sobre la que los empresarios pueden establecer una actividad social o comercial (Comisión Europea, 2006: 4).

Siguiendo esta definición, en el Informe Final sobre la Iniciativa Emprendedora publicado en el 2008, se estableció que siendo conscientes del importante papel que juega la educación en la promoción de actitudes y comportamientos emprendedores, se ha de considerar que la iniciativa emprendedora es "una competencia clave para todos, que ayuda a los jóvenes a ser más creativos y tener más confianza en sí mismos en cualquier actividad que emprendan" (Comisión Europea, 2008: 4).

De hecho, el sentido de la iniciativa y espíritu de empresa es una de las competencias clave del marco europeo ${ }^{2}$ y una acción de la Comunicación de la Comisión sobre Un nuevo concepto de Educación (Comisión Europea, 2013b). Así es que en esta Comunicación, la Comisión insiste en que la inversión en educación y forma-

2. Según la Recomendación 2006/962/CE del Parlamento Europeo y del Consejo, de 18 de diciembre de 2006, sobre las competencias clave para el aprendizaje permanente (Diario Oficial L 394 de 30.12.2006), se entiende por sentido de la iniciativa y espíritu de empresa "la habilidad de transformar las ideas en actos y que está relacionado con la creatividad, la innovación y la asunción de riesgos, así como con la habilidad para planificar y gestionar proyectos con el fin de alcanzar objetivos. Las personas son conscientes del contexto en el que se sitúa su trabajo y pueden aprovechar las ocasiones que se les presenten. El sentido de la iniciativa y el espíritu de empresa son el fundamento para la adquisición de cualificaciones y conocimientos específicos necesarios para aquellos que crean algún tipo de actividad social o comercial o que contribuyen a ella. Dicho espíritu debería comportar asimismo una concienciación sobre los valores éticos y fomentar la buena gobernanza." 
ción son fundamentales para impulsar el crecimiento y la competitividad. Subraya que hay que concentrar esfuerzos en el desarrollo de habilidades (competencias) transversales como el pensamiento crítico, la iniciativa, la solución de problemas y el trabajo colaborativo porque preparan a las personas para los recorridos de su carrera profesional, que son hoy en día tan variados e impredecibles. Así mismo, apuesta por las competencias las relacionadas con el emprendimiento porque éstas no sólo contribuyen a la creación de nuevas empresas, sino también a la empleabilidad de los jóvenes.

En definitiva, emprendimiento, iniciativa emprendedora, espíritu emprendedor o competencia emprendedora, lo que ha de quedar suficientemente justificado es la necesidad de promover e implementar una educación en emprendimiento que comience con la puesta en marcha de programas formativos dirigidos a la sociedad en general y a los futuros maestros en particular. Al fin y al cabo, éstos serán modelos de referencia importantes para las nuevas generaciones de ciudadanos de una sociedad en constante cambio que demanda personas y profesionales competentes y capaces de dar respuesta, de manera innovadora, a los distintos retos que se aproximan.

En el caso de los futuros maestros, no tenemos más que fijarnos en las funciones que se le vienen otorgando al profesor desde la Ley Orgánica 2/2006 (artículo 91). En esta Ley se establece que son propias del profesor la orientación profesional de los alumnos, la atención al desarrollo social de los mismos, el fomento de valores de una ciudadanía democrática o la mejora continua de su profesión docente. Más aún, no hay más que acercarse al borrador del nuevo decreto por el que se establece el currículo de la Educación Básica y se implanta en la Comunidad Autónoma del País Vasco (también conocido como Heziberri), para darnos cuenta del valor que añadiría a la formación del futuro maestro una formación en emprendimiento.

De hecho, en el borrador de Heziberri se subraya que, entre las acciones formativas promovidas por el departamento competente en materia educativa se promoverá la innovación mediante el análisis y reflexión sobre la práctica docente; se formará profesionales con responsabilidad social, críticos pero proactivos y creativos ante los cambios; se promoverá la excelencia mediante el impulso de proyectos para la innovación educativa; y, se favorecerán procesos de colaboración entre los diferentes profesionales (formación entre iguales, creación de redes).

Así mismo, en este mismo borrador, se aboga por un perfil competencial que permita a los maestros asumir en condiciones óptimas las responsabilidades propias de sus diferentes funciones y tareas. Entre ellas destacamos enseñar los procedimientos y actitudes para que el alumnado aprenda a emprender; investigar e innovar los procesos de enseñanza-aprendizaje; cooperar con otros profesores y profesoras y agentes educativos y participar en la gestión y mejora continua del centro educativo; desarrollar una visión ética sobre su profesión y su compromiso social como agente innovador; $y$, resolver problemas y conflictos.

Finalmente, para ello se estima necesario que la formación del maestro se apoye en la puesta en marcha de la innovación en los centros, y en la cooperación, la interacción entre iguales, el trabajo en equipo y el compromiso compartido para lograr la mejora de la práctica educativa. Siendo el fin último favorecer el progreso hacia una comunidad educadora. 
En definitiva, se trataría de ver en la formación en emprendimiento un aliado perfecto para la adecuada formación del futuro maestro como motor de cambio y agente social innovador de primera calidad (Hattie, 2003; y Berry, Rasberry y WiIliams, 2007).

\section{Objetivos de la educación emprendedora}

Una vez asumida la importancia y necesidad de una educación en emprendimiento, el siguiente paso es definir el objetivo de la misma. Según el Informe Final sobre la Iniciativa Emprendedora, el principal objetivo de la educación emprendedora debería ser el desarrollo de las capacidades, habilidades y mentalidad emprendedora, siendo objetivos más específicos los siguientes:

... desarrollar el dinamismo emprendedor y la iniciativa entre los estudiantes (por medio de la sensibilización y la motivación); proporcionar a los estudiantes los conocimientos necesarios para fundar una empresa y gestionar su crecimiento; y desarrollar las habilidades emprendedoras necesarias para identificar y explotar las oportunidades de negocio (Comisión Europea, 2008: 4).

Pero no sólo eso, en este Informe se subraya la necesidad de que los programas y cursos sobre iniciativa emprendedora permitan a los estudiantes (además de ser los protagonistas del proceso de enseñanza-aprendizaje): ser más creativos o innovadores; tener un alto nivel de motivación; ser proactivos y conscientes de sus capacidades; tener confianza en sí mismos; estar dispuestos a aceptar desafíos; ser mejores comunicadores, estrategas, líderes y negociadores; trabajar mejor en red; resolver mejor los problemas; trabajar mejor en equipo y pensar de forma sistemática; ser más independientes, menos reacios a arriesgarse y capaces de vivir con incertidumbre y de reconocer las oportunidades.

\section{Planteamientos metodológicos adecuados para desarrollar la iniciativa empren- dedora e implementar la educación en emprendimiento}

En el Plan 2020 se insiste en la necesidad de que "la educación debe estar ligada a la realidad mediante modelos prácticos de aprendizaje basados en la experiencia y también en la experiencia de los emprendedores del mundo real" (Comisión Europea, 2013a: 6). Para ello será preciso "definir los resultados del aprendizaje del emprendimiento para todos los formadores a fin de introducir en las aulas metodologías eficaces" (Comisión Europea, 2013a: 6).

En esta misma línea argumental, la Comisión añade que la mentalidad y competencias emprendedoras pueden ser desarrolladas de manera efectiva a través de la práctica, las experiencias de la vida real y el trabajo por proyectos. También insiste en que éstas competencias deberían ser implementadas en todas las asignaturas, bien sea como asignatura independiente o de manera interdisciplinar, siendo especialmente importante tanto para los intra-emprendedores como para los líderes e innovadores de un grupo u organización. Pero lo que más se subraya es que dichas competencias deberán ser promovidas, además de por las instituciones educativas, las empresas y la comunidad en general. 
Por su parte, en la Comunicación de la Comisión sobre Un nuevo concepto de Educación se insiste en que los Estados miembros deben:

... estimular las aptitudes emprendedoras a través de maneras de enseñar y aprender nuevas y creativas desde la educación primaria, además de prestar una atención particular, desde la enseñanza secundaria hasta la superior, a la oportunidad de crear empresas como objetivo de carrera. Con un aprendizaje basado en problemas concretos y relaciones con las empresas, la experiencia del mundo real ha de integrarse en todas las disciplinas y adaptarse a todos los niveles de la educación. Todos los jóvenes deben beneficiarse de una experiencia de emprendimiento práctica como mínimo antes de abandonar la enseñanza obligatoria (Comisión Europea, 2013b: 17).

Lo que ha de quedar claro es que las clases magistrales no son los métodos de enseñanza más adecuados para desarrollar una mentalidad emprendedora sino que es el uso de métodos de enseñanza basados en la experiencia los que son cruciales para el desarrollo de competencias emprendedoras (Arruti, 2011, 2012 y 2013; y Vainio, Heinone, Veripää y Varis, 2005). En esa misma línea se aboga por la utilización de enfoques de aprendizaje más interactivos, donde el profesor sea más un moderador y no tanto un conferenciante.

En el Informe Final sobre la Iniciativa Emprendedora, además de recomendar a los formadores la necesidad de buscar un equilibrio entre los aspectos teóricos y prácticos de la enseñanza, se propone el uso de métodos interactivos y pragmáticos, autoaprendizaje activo, pedagogías orientadas a la acción, dinámicas de grupo, trabajo en equipo, estudios de caso, aprendizaje a través de proyectos, métodos centrados en los estudiantes, talleres, celebración de conferencias, invitación de expertos, simulaciones, aprendizaje por medio de experiencias directas, métodos de desarrollo personal y autoevaluación.

Por su parte, el proyecto de la Unión Europea CIP-Project Young Entrepreneurship Developing in Action (CIP-Proyecto Joven Emprendedor en Desarrollo en Acción) encaminado a servir de apoyo a los futuros maestros, subraya como experiencias de aprendizaje efectivas para el desarrollo de las competencias de emprendimiento las que pasamos a nombrar a continuación ${ }^{3}$ : proyectos de aprendizaje, aprendizaje activo, aprendizaje exploratorio, aprendizaje experiencial, aprendizaje en entornos reales y auténticos, aprendizaje colaborativo, aprendizaje productivo, creación de valor, aprendizaje emprendedor, aprendizaje a partir de las propias ideas, aprendizaje a partir del fracaso y la tolerancia, aprendizaje a partir del error. Se trata de que el maestro sea capaz de adaptar los entornos de aprendizaje y sirva de guía a los estudiantes.

Alemany, Marina y Pérez Días-Pericles (2013) destacan las siguientes metodologías como las que se están manejando con más fuerza a la hora de desarrollar las competencias: aprendizaje cooperativo, aprendizaje asistido por otros estudiantes, aprendizaje asistido por la enseñanza a otros, aprendizaje basado en problemas, aprendizaje basado en proyectos, aprendizaje por investigación/indagación, aprendizaje integrado por el trabajo, aprendizaje reflexivo, aprendizaje holístico, aprender a pensar y aprendizaje servicio, ésta última como metodología activa con sello de garantía.

3. Véase YEDAC-project: http://www.yedac.eu/media/3891/Teacher-guidelines-140924.pdf o entre en la página: http://www.yedac.eu/. 
En concreto, estos autores proponen algunas recomendaciones metodológicas en relación a la competencia aprender a emprender, que permitirían entrenar y evaluar algunos de los desempeños considerados básicos para el desarrollo de la competencia. A continuación destacamos las siguientes:

- utilizar la heteroevaluación, coevaluación y la autoevaluación con el fin de promover, entre otros, la identificación de fortalezas y debilidades, la motivación de logro y el espíritu de superación;

- estructurar la docencia en el aula de manera democrática, para ayudar a los participantes a tomar conciencia de su autonomía y su capacidad de decisión;

- informar y analizar las causas de éxito o fracaso obtenido, incidiendo en las razones, reflexionando sobre ellas y dando oportunidades para rectificar y mejorar;

- fomentar el trabajo cooperativo a través del trabajo en equipo y establecimiento de roles rotativos, promoviendo un liderazgo democrático;

- proponer actividades adaptadas a los intereses y habilidades de los participantes, y que sean, a su vez, actividades desafiantes, retantes y que estimulen la motivación de logro;

- incidir en la motivación intrínseca y en la importancia del esfuerzo para la obtención del éxito;

- utilizar el modelado como estrategia metodológica;

- estimular la creatividad, el fomento de la inventiva y la generación de ideas;

- potenciar la generación de actividades que fomenten la presentación de juicios y valoraciones diferentes;

- incitar a la comunicación activa a través de la realización de preguntas divergentes;

- ayudar a los alumnos a marcarse metas, registrar progresos, planificar y evaluar proyector con el fin de adquirir hábitos de trabajo que favorezcan la consecución de los objetivos fijados.

\section{EL PROFESOR EMPRENDEDOR O TEACHERPRENEUR}

Siguiendo la estela marcada por el Plan 2020 y la Comunicación Un nuevo concepto de Educación, la Comisión publica en 2014 la guía Educación en emprendimiento: Guía del educador. En ella se vuelve a reconocer la importancia del papel que desempeña el profesor en el desarrollo de la actitud emprendedora de sus alumnos. No en vano, los profesores son considerados facilitadores del aprendizaje y multiplicadores de ideas, quienes pueden moldear los procesos de aprendizaje de los alumnos y ayudarles a conseguir el desarrollo de competencias relacionadas con el emprendimiento. Tanto es así que la Comisión reconoce la necesidad de dotar a los profesores de una amplia gama de competencias relacionadas con la creatividad y el emprendimiento, así como de un entorno escolar adecuado en el que se fomenten la creatividad y la asunción de riesgos y donde los errores se consideren una oportunidad de aprendizaje. Y lo que es más importante, subraya la necesidad de que 
"todo profesor y futuro profesor ha de acceder durante su carrera a una experiencia de formación en los temas y métodos clave relativos al aprendizaje emprendedor y la educación en emprendimiento" (Comisión Europea, 2014: 7) porque lo que está claro es que difícilmente van a poder guiar los profesores a sus alumnos en la tarea de ser emprendedores si ellos mismos no lo son.

\section{Características del teacherpreneur}

Según la Comisión Europea (2014) y autores como Barnett, Byrd y Wieder (2013) o Berry (2011), los teacherpreneurs son profesores que poseen una pasión por enseñar, que son fuente de inspiración, que tienen mentalidad abierta y son seguros de sí mismos, que son flexibles y, a la vez, responsables, pero también personas que se atreven a romper de vez en cuando las reglas preestablecidas. Se trata de personas que saben escuchar, aprovechar y vender nuevas ideas, trabajar con el alumnado (siempre orientando su trabajo a la acción). Son grandes jugadores de equipo con una importante y extensa red de contactos profesionales (networking). No se cierran en sus aulas sino que se abren al exterior e incluyen a expertos en su quehacer diario en las aulas, centrando el proceso de aprendizaje en experiencias de la vida real. Así mismo, como buenos profesionales, suelen seguir un plan de trabajo (con sus correspondientes programaciones). Son personas flexibles y adaptables, y abogan por un aprendizaje interdisciplinar, que se basa en el ABP (Aprendizaje Basado en Proyectos) y en el que el libro de texto suele ser sustituido por materiales y recursos que ellos mismos diseñan y/o se encargan de preparar. Son profesores que ponen énfasis en los procesos e interacciones que tienen lugar entre personas que pertenecen a un grupo, y son conscientes de que en sus clases sí hay lugar para la diversidad (de opiniones, de respuestas, de soluciones) y para la reflexión sobre el proceso de aprendizaje. En definitiva, un teacherpreneur es más que una persona que enseña su lección de manera magistral, es un acompañante, un guía, una persona que se centra en los procesos de aprendizaje de cada alumno y en el desarrollo de sus competencias.

El anteriormente citado proyecto de la Unión Europea CIP-Project Young Entrepreneurship Developing in Action destaca entre las competencias emprendedoras (Sáenz y López, 2015) (definidas como conocimientos sobre el emprendimiento y la educación en emprendimiento, actitud reflexiva abierta hacia el emprendimiento) que los profesores han de desarrollar en el aula y a lo largo de su proceso de desarroIlo profesional, las siguientes competencias:

- Competencias emprendedoras: El profesor puede identificar y aprovechar las oportunidades; hacer red; organizar y planificar actividades; idear un plan; tomar decisiones; hacer uso de la experiencia; tratar de crear valor y calidad; asumir la responsabilidad de una actividad; tomar riesgos; crear nuevas ideas; actuar de forma innovadora; convertir las ideas en acciones; dedicarse a una actividad; hacer esfuerzos para lograr los objetivos de un actividad; presentar ideas y productos y apoyar el desarrollo de habilidades de gestión de proyectos.

- Competencias personales: El profesor es capaz de gestionar conflictos; cooperar en equipos; comunicarse con otros; dar consejos; dar apoyo en el desarrollo del proceso de aprendizaje personal; reflexionar sobre su propio papel 
como maestro; identificar sus fortalezas y capacidades; manejar errores de forma constructiva; generar ideas para resolver problemas; comprender y valorar otras ideas; argumentar a favor o en contra de las ideas o productos; ayuda a alcanzar consenso; desarrollar una conciencia cultural y social; plantear y discutir temas éticos; y actuar y reaccionar de manera flexible.

- Competencias didácticas: El profesor puede definir competencias para la educación en emprendimiento; planificar entornos de aprendizaje para la educación en emprendimiento; organizar actividades de aprendizaje en el marco de la educación en emprendimiento; integrar la educación en emprendimiento en las asignaturas a enseñar; evaluar actividades de aprendizaje; reflexionar sobre actividades de enseñanza; mejorar actividades de enseñanza; cambiar mentalidades; motivar a los estudiantes; alentar ideas / talentos e intereses de los estudiantes; crear un apoyo continuo para el desarrollo de la competencia espíritu emprendedor; promover la creatividad de los estudiantes; facilitar y guiar el trabajo de los estudiantes; optimizar el uso de recursos; diagnosticar habilidades de los estudiantes; y configurar entornos inclusivos de aprendizaje (YEDAC-project: 6-7).

La adopción de un aprendizaje basado en competencias obliga a un cambio de las funciones del profesor puesto que éste pasa de ser un "mero" proveedor o transmisor de conocimiento a convertirse en un facilitador del aprendizaje. Nos referimos a una especie de mentor (coach) que guía los procesos de aprendizaje de los estudiantes no sólo de manera individual sino también como miembros de una comunidad que aprende. En ese contexto, el profesor es el que desafía al estudiante al proponer situaciones problemáticas que admiten más de una solución y a utilizar técnicas de evaluación distintas a los tradicionales exámenes escritos (Villa y Poblete, 2008; y YEDAC-project).

Las características de los teacherpreneurs (Comisión Europea, 2014) coinciden con las que Alemany, Alvarez, Planellas y Urbano (2011) y Alemany y Planellas (2011) consideran como propias de un emprendedor, es decir, la necesidad de logro, la independencia, el locus de control interno, esto es, la percepción de que los resultados son producidos por las acciones propias, la propensión al riesgo, la auto-confianza, la creatividad y la iniciativa, y que coinciden con la personalidad del emprendedor. También guardan similitudes con los indicadores en que desglosan la competencia aprender a emprender Alemany, Marina y Pérez Días-Pericles (2013), esto es: autonomía personal, liderazgo, innovación y habilidades empresariales, y con los valores del espíritu emprendedor considerados indispensables y propuestos por la Dirección General de Política de la Pequeña y Mediana Empresa (2003). En este último caso destacan los valores personales (creatividad, autonomía, confianza en uno mismo, tenacidad, sentido de la responsabilidad y capacidad para asumir riesgos) y los sociales (liderazgo, espíritu de equipo y solidaridad).

\section{Claves para el diseño y desarrollo de programas de educación en EMPRENDIMIENTO PARA FUTUROS MAESTROS}

Llegado a este punto nos atrevemos a decir que no nos cabe ninguna duda de que uno de los objetivos de la formación de los futuros maestros es lograr que la compe- 
tencia emprendedora forme parte del currículum del grado en Educación Primaria. De hecho, no en vano la Ley 14/2013, de 27 de septiembre, de apoyo a los emprendedores y su internacionalización, dedica sus artículos 4, 5 y 6 del capítulo I del TÍTULO I (Apoyo a la iniciativa emprendedora) a la Educación en emprendimiento.

En el artículo 4 sobre el emprendimiento en la enseñanza primaria y secundaria, en lo que al currículum de Educación Primaria se refiere, se establece que se deberán incorporar objetivos, competencias, contenidos y criterios de evaluación de la formación orientados al desarrollo y afianzamiento del espíritu emprendedor. Así mimo, se estima necesario proponer medidas para que el alumnado participe en actividades que le permitan afianzar el espíritu emprendedor y la iniciativa empresarial a partir de aptitudes como la creatividad, la iniciativa, el trabajo en equipo, la confianza en uno mismo y el sentido crítico.

Por su parte, en el artículo 6 sobre formación del profesorado en materia de emprendimiento se apunta que los profesores deberán adquirir las competencias y habilidades relativas al emprendimiento, la iniciativa empresarial, la igualdad de oportunidades en el entorno empresarial, y la creación y desarrollo de empresas. Todo ello a través de la formación inicial o de la formación permanente del profesorado.

En el caso de la Ley Orgánica 8/2013, de 9 de diciembre, para la mejora de la calidad educativa (en adelante, LOMCE), se establece que una sociedad más abierta, global y participativa como la nuestra demanda un perfil de personas y profesionales más activos, sofisticados, diversificados, con autoconfianza, curiosas, emprendedoras e innovadoras. En definitiva, personas deseosas de participar a través de procesos colaborativos en la sociedad a la que pertenecen, de crear valor individual y colectivo, y capaces de asumir como propio el valor del equilibrio entre el esfuerzo y la recompensa. Para ello, y con el fin de estimular el espíritu emprendedor de los participantes, en su artículo 17 (letra b) la LOMCE establece la necesidad de desarrollar hábitos de trabajo individual y de equipo, de esfuerzo y de responsabilidad en el estudio, así como actitudes de confianza en sí mismo, sentido crítico, iniciativa personal, curiosidad, interés y creatividad en el aprendizaje, y espíritu emprendedor. El papel de los profesores será esencial, sin duda, para el logro de dicho objetivo.

La anterior Ley Orgánica 2/2006, de 3 de mayo, de Educación ya contemplaba entre sus fines (artículo 2, letra f) el desarrollo de la capacidad de los alumnos para regular su propio aprendizaje así como para desarrollar la creatividad, la iniciativa personal y el espíritu emprendedor. Por su parte, el artículo 17 (Objetivos de la educación primaria), destacaba la letra b que apuntaba al desarrollo de hábitos de trabajo individual y de equipo, de esfuerzo y responsabilidad en el estudio, así como actitudes de confianza en sí mismo, sentido crítico, iniciativa personal, curiosidad, interés y creatividad en el aprendizaje.

El Decreto 175/2007, de 16 de octubre, por el que se establece el currículo de la Educación Básica y se implanta en la Comunidad Autónoma del País Vasco destaca en su artículo 6, letra f (Competencias educativas generales) como uno de los grandes ejes referenciales para una educación integral: aprender a hacer y a emprender, teniendo iniciativa para tomar decisiones y asumir responsabilidades, valorando el esfuerzo y la superación de las dificultades y practicando iniciativas emprendedoras en los diferentes ámbitos de la vida. Por otra parte, en su artículo 7, letra h (Com- 
petencias básicas) señala la competencia para la autonomía e iniciativa personal. Y finalmente en lo que se refiere a los objetivos de la Educación Básica, concretamente a la competencia (aprender a hacer y emprender) (artículo 8), destacan la letra a (desarrollar el espíritu emprendedor mediante actitudes de confianza en uno mismo, la participación, el sentido crítico, y la iniciativa personal, comprometiéndose en proyectos que requieran planificar, tomar decisiones y asumir responsabilidades) y la letra b (identificar las aptitudes propias, y valorar los aspectos positivos y negativos de cada una de las distintas opciones de estudios y trabajo, para definir su proyecto personal académico y profesional con realismo y de manera adecuada a sus capacidades y valores).

Tabla 1. Recomendaciones para el diseño de acciones formativas

\begin{tabular}{|c|c|c|c|}
\hline Competencias & Contenidos & $\begin{array}{l}\text { Métodos pedagógicos } \\
\text { innovadores }\end{array}$ & Sistemas de evaluación \\
\hline $\begin{array}{l}\text { Liderazgo } \\
\text { Audacia } \\
\text { Iniciativa } \\
\text { Perseverancia } \\
\text { Disposición, asunción y } \\
\quad \text { gestión de riesgos } \\
\text { Tolerancia a la incertidumbre } \\
\text { Aceptación del fracaso } \\
\text { Toma de decisiones } \\
\text { Innovación } \\
\text { Creatividad } \\
\text { Pensamiento creativo } \\
\text { Pensamiento sistémico } \\
\text { Pensamiento crítico } \\
\text { Confianza en uno mismo } \\
\text { Reflexión } \\
\text { Orientación al aprendizaje } \\
\text { Capacidades de negociación } \\
\text { Gestión de proyectos } \\
\text { Búsqueda de información } \\
\text { Búsqueda de oportunidades }\end{array}$ & $\begin{array}{l}\text { Concepto de profesor } \\
\text { entrenador y profesor } \\
\text { facilitador } \\
\text { Concepto de evaluación } \\
\text { integral } \\
\text { Concepto de ciudadanía } \\
\text { participativa } \\
\text { Concepto de flexibilidad } \\
\text { Las TIC y los nuevos medios de } \\
\text { comunicación } \\
\text { Generación, valoración y } \\
\text { selección de acciones } \\
\text { educativas innovadoras } \\
\text { Las innovaciones educativas } \\
\text { Importancia en la implicación } \\
\text { en los problemas locales } \\
\text { El compromiso (social) } \\
\text { Innovaciones educativas } \\
\text { Importancia de las redes } \\
\text { educativas y el trabajo } \\
\text { en red }\end{array}$ & $\begin{array}{l}\text { Aprendizaje basado en la } \\
\text { competencia y el talento } \\
\text { Aprendizaje basado en la } \\
\text { experiencia } \\
\text { Talleres } \\
\text { Aprendizaje práctico } \\
\text { Práctica profesional } \\
\quad \text { colaborativa (eficaz) } \\
\text { Trabajo en equipo } \\
\text { Trabajo por proyectos } \\
\text { Sentido de la visión y la } \\
\text { misión } \\
\text { Responsabilidad de los } \\
\text { estudiantes en sus propios } \\
\text { procesos de aprendizaje } \\
\text { Itinerarios de aprendizaje } \\
\text { flexible } \\
\text { Aprendizaje colaborativo } \\
\text { Resolución de problemas } \\
\text { Visitas a empresas } \\
\text { Testimonios de expertos } \\
\text { Estudios de caso } \\
\text { Experimentación } \\
\text { Simulaciones } \\
\text { Laboratorios } \\
\text { Investigación-acción } \\
\text { Debate, diálogo e interacción } \\
\text { Asociacionismo } \\
\text { Aprendizaje a partir del error }\end{array}$ & $\begin{array}{l}\text { Elaboración de materiales } \\
\text { Elaboración de diccionarios de } \\
\text { emprendimiento } \\
\text { Diseño de acciones de } \\
\text { emprendimiento } \\
\text { Diseño de metodologías de } \\
\text { aprendizaje innovadoras } \\
\text { Elaboración de nuevos } \\
\text { productos o servicios } \\
\text { a utilizar en el ámbito } \\
\text { educativo } \\
\text { Implementación de acciones } \\
\text { formativas innovadoras } \\
\text { Participación en concursos } \\
\text { Coevaluación }\end{array}$ \\
\hline
\end{tabular}

Nota: elaboración propia. 
Ante esta situación nos preguntamos sobre cuál será la propuesta de formación en la competencia emprendedora para maestros de Educación Primaria. Si bien ignoramos la respuesta, tras analizar los casos de buenas prácticas presentados en la Guía del Educador (Comisión Europea, 2014), presentamos en la tabla 1 lo que consideramos pudieran ser las principales aportaciones en cuanto a aspectos clave a considerar a la hora de diseñar acciones formativas orientadas a desarrollar la competencia emprendedora en los currículums del grado en Educación Primaria.

A este respecto, y con el fin de ayudar en el desarrollo de procesos de aprendizaje eficaces, el proyecto de la Unión Europea CIP-Project Young Entrepreneurship Developing in Action tiene como objetivo servir de apoyo a los maestros en el desarrollo de entornos de aprendizaje y procesos encaminados a lograr la educación en emprendimiento en el sentido más amplio del término, y ofrecen un modelo didáctico y un modelo de proceso de aprendizaje.

\section{REFLEXIONES fiNALES A MODO DE CONCLUSIÓN}

El objetivo de este artículo era triple. Por una parte incidir en la importancia que el emprendimiento y la educación en emprendimiento tienen para una sociedad tan competitiva como la nuestra. En segundo lugar, profundizar en el concepto de teacherpreneur así como en las características y competencias que le son propias. Y en tercer lugar, realizar una serie de recomendaciones sobre las competencias que el futuro maestro de Educación Primaria debería desarrollar durante el proceso de obtención del grado en Educación Primaria.

Si bien creemos que hemos logrado los objetivos propuestos, quisiéramos plantear una serie de conclusiones a modo de cierre. La primera conclusión a la que llegamos tras el análisis y reflexión realizados es que consideramos que, a nuestro parecer, queda más que demostrada la importancia que la competencia en emprendimiento supone para el futuro maestro de primaria. Esta conclusión se basa principalmente en las competencias propias del teacherpreneur que definen su perfil profesional y personal, y que han sido expuestas previamente (auto-confianza, creatividad, iniciativa, innovación, necesidad de logro, autonomía y liderazgo, entre otras). El desarrollo por parte del maestro de primaria de dichas competencias es fundamental para poder llevar a la práctica adecuadamente su trabajo docente.

Igual de importante es considerar la competencia aprender a emprender que los niños de educación primaria han de desarrollar durante su escolarización obligatoria. Porque, ¿cómo lograrlo si los maestros, uno de los grandes facilitadoras de su aprendizaje, no cuentan con ellas? Siempre es mejor contar con profesionales bien formados en todos los ámbitos.

La segunda conclusión a la que llegamos es que es obvio que existe la necesidad de diseñar y desarrollar acciones educativas de diversa índole encaminadas al desarrollo de la competencia emprendedora entre los alumnos de los grados de Educación Primaria. Insistimos en que serán ellos los futuros maestros, las personas de referencia para los niños que cada año pasan por las aulas de Educación Primaria con el fin de desarrollarse como personas y miembros de una sociedad cambiante y desafiante. No se trata de proponer una asignatura sobre emprendimiento sino de 
ponernos a idear y crear el itinerario más adecuado para que los futuros maestros sean ejemplo y referencia no sólo para los alumnos sino también para profesionales de otros ámbitos. Nuestra sociedad lo agradecerá.

Finalmente, sin querer desmerecer ningún trabajo, pues somos conscientes de lo mucho que supone diseñar y desarrollar un currículum, y conscientes de que aún queda mucho por hacer, desde aquí nos comprometemos a diseñar una mención para el desarrollo del teacherpreneur, una mención que se base en el desarrollo de la competencia emprendedora y que ponga el acento en las características básicas del teacherpreneur, uno de los grandes protagonistas del proceso de enseñanza-aprendizaje junto con nuestros alumnos, emprendedores en potencia.

Porque aunque estamos convencidos de que no empezamos de cero, sí que creemos que es necesario replantearse el marco de actuación (las competencias consideradas genéricas y específicas consideradas básicas para la formación del maestro) y, sobre todo, el modo de actuación (metodologías de enseñanza-aprendizaje utilizadas) así como los sistemas y herramientas de evaluación que estamos manejando en la actualidad. Ha llegado el momento de poner en práctica nuestra propia competencia emprendedora.

\section{REFERENCIAS BIBLIOGRÁFICAS}

Arruti, A. (2011). Programa de emprendizaje Ingenio. En Gabinete de Rectorado y de Comunicación URL (Coord.), Jornadas Interuniversitarias de innovación docente 2011 (pp. 151-158). Barcelona: Universitat Ramón Llul.

Arruti, A. (2012). EKin-IT. An entrepreneurial programme developed at the University of Deusto. I am also an entrepreneur. 5th International Conference of Education, Research and Innovation. ICERI 2012. Abstracts CD. Madrid (Spain).

Arruti, A. (2013). The power of Ekin-IT to empower entrepreneurs, an innovative initiative from the Deusto Entrepreneurship Centre (University of Deusto). The 7th International Technology, Education and Development Conference. INTED 2013. Proceedings CD. Valencia (Spain).

Alemany, L., Marina, J.A., y Pérez Días-Pericles, J.M. (2013). Aprender a emprender. Cómo educar el talento emprendedor. Barcelona: Aula Planeta.

Alemany, L. Álvarez, C., Planellas, M., y Urbano, D. (2011). Libro blanco de la iniciativa emprendedora en España. Barcelona: ESADE.

Alemany, L., y Planellas, M. (2011). Emprender es posible. Barcelona: Planeta.

Barnett, B., Byrd, A., y Wieder, A. (2013). Teachepreneurs. Innovative teachers who lead but don't leave. San Francisco: Jossey-Bass.

Berry, B. (2011). Teaching 2030: What we must do for our students and our public schools... now and in the future. New York: Teachers College Press.

Berry, B., Rasberry, M., y Williams, A. (2007). Recruiting and retraining quality teachers for high needs schools: Insights from NBTS summits and others policy initiatives. Carrboro: Center for Teaching Quality.

Comisión de las Comunidades Europeas (2003). Libro verde. El espíritu empresarial en Europa. Recuperado de http://www.oei.es/etp/green_paper_final_es.pdf. 
Comisión Europea (2004). Implementation of "Education and Training 2010". Work programme. Recuperado de https://goo.gl/FTca3e.

Comisión Europea (2006). Aplicar el programa comunitario de Lisboa: Fomentar la Mentalidad Emprendedora mediante la Educación y la Formación. Recuperado de http://goo.gl/tUL7zX.

Comisión Europea (2008). La Iniciativa Emprendedora en la Enseñanza Superior, especialmente en Estudios no Empresariales. Resumen del informe final del grupo de expertos. Recuperado de http://www.cdiex.org/aprendizaje/documento_8.pdf.

Comisión Europea (2012). Comunicado de Prensa de la Comisión Europea. Recuperado de http://europa.eu/rapid/press-release_IP-12-1224_es.htm.

Comisión Europea (2013a). Plan de acción sobre emprendimiento 2020. Recuperado de http://ec.europa.eu/transparency/regdoc/rep/1/2012/ES/1-2012-795-ES-F1-1.Pdf.

Comisión Europea (2013b). Un nuevo concepto de educación: invertir en las aptitudes para lograr mejores resultados socioeconómicos. Recuperado de http:// ec.europa.eu/transparency/regdoc/rep/1/2012/ES/1-2012-669-ES-F2-1.Pdf.

Comisión Europea (2014). La educación en emprendimiento. Guía del educador. Recuperado de http://goo.gl/fHZjpr.

Decreto 175/2007, de 16 de octubre, por el que se establece el currículo de la Educación Básica y se implanta en la Comunidad Autónoma del País Vasco. Recuperado de: http://www.euskadi.eus/bopv2/datos/2007/11/0706182a.pdf.

Dirección General de Política de la Pequeña y Mediana Empresa y Secretaría General (2003). El espíritu emprendedor. Motor de futuro. Guía del profesor. Madrid: Ministerio de Economía.

Gibb, A., Haskins, G., y Robertson, I. (2013). Leading the Entrepreneurial University: Meeting the Entrepreneurial Development Needs of Higher Education Institutions. En A. Altmann y B. Ebersberger (Eds.), Universities in Change, Innovation, Technology, and Knowledge Management (pp. 9-45). New York: Springer. doi: 10.1007/978-1-4614-4590-6_2.

González, J., y Wagenaar, R. (2003). Tuning Educational Structures in Europe. Bilbao: Universidad de Deusto.

Hattie, J. (2003). Teachers make a difference: What is the research evidence? Camberwell: Australian Council for Educational Research.

Ley 14/2013, de 27 de septiembre, de apoyo a los emprendedores y su internacionalización. Recuperado de https://www.boe.es/boe/dias/2013/09/28/pdfs/BOEA-2013-10074.pdf.

Ley Orgánica 8/2013, de 9 de diciembre, para la mejora de la calidad educativa. Recuperado de http://www.boe.es/boe/dias/2013/12/10/pdfs/BOE-A-2013-12886.pdf.

Ley Orgánica 2/2006, de 3 de mayo, de Educación. Recuperado de http://www.boe. es/boe/dias/2006/05/04/pdfs/A17158-17207.pdf.

Marina, J.A. (2010). La competencia de emprender. Revista de Educación, 351, 49-71.

Recomendación 2006/962/CE del Parlamento Europeo y del Consejo, de 18 de diciembre de 2006, sobre las competencias clave para el aprendizaje permanente (Diario Oficial L 394 de 30.12.2006). 
Sáenz, N., y López, A.L. (2015). Las competencias de emprendimiento social, COEMS: Aproximación sobre los programas de formación universitaria de lberoamérica. REVESCO. Revista de Estudios Cooperativos, 118.

Taatila, V.P. (2010). Learning entrepreneurship in higher education. Education + Training, 52(1), 48-61.

Vainio, S., Heinone, P., Veripää, S., y Varis, L. (2005). El espíritu emprendedor y la formación: el caso Team Academy. En Diputación Foral de Gipuzkoa y Cluster de Conocimiento (Orgs.), Cómo crear capacidades de aprendizaje y emprendizaje (pp. 48-53). Zamudio: PMP.

Villa, A., y Poblete, M. (2008). Aprendizaje basado en competencias. Una propuesta para la evaluación de las competencias genéricas. Bilbao: Universidad de Deusto. 